ACCepted for publication By the Astrophysical Journal

Preprint typeset using $\mathrm{AT}_{\mathrm{E} X} \mathrm{X}$ style emulateapj v. 01/23/15

\title{
EFFECT OF TEMPLATE UNCERTAINTIES ON THE WMAP AND Planck MEASURES OF THE OPTICAL DEPTH DUE TO REIONIZATION
}

\author{
J. L. Weiland, K. Osumi, G. E. Addison, C. L. Bennett, D. J. Watts \\ Department of Physics and Astronomy, Johns Hopkins University, 3400 N. Charles St, Baltimore, MD 21218, USA \\ AND \\ M. Halpern, G. Hinshaw \\ Department of Physics and Astronomy, University of British Columbia, Vancouver, BC, V6T 1Z1, Canada \\ Accepted for publication by the Astrophysical Journal
}

\begin{abstract}
The reionization optical depth is the most poorly determined of the six $\Lambda$ CDM parameters fit to CMB anisotropy data. Instrumental noise and systematics have prevented uncertainties from reaching their cosmic variance limit. At present, the datasets providing the most statistical constraining power are the WMAP, Planck LFI, and Planck HFI full-sky polarization maps. As the reprocessed HFI data with reduced systematics are not yet publicly unavailable, we examine determinations of $\tau$ using 9year WMAP and 2015 Planck LFI data, with an emphasis on characterizing potential systematic bias resulting from foreground template and masking choices. We find evidence for a low-level systematic in the LFI polarization data with a roughly common-mode morphology across the LFI frequencies and a spectrum consistent with leakage of intensity signal into the polarization channels. We demonstrate significant bias in the optical depth derived when using the LFI $30 \mathrm{GHz}$ map as a template to clean synchrotron from WMAP data, and recommend against the use of the 2015 LFI $30 \mathrm{GHz}$ polarization data as a foreground template for non-LFI datasets. We find an inconsistency between versions of the 2015 polarized $353 \mathrm{GHz}$ dust templates reconstructed from the Planck likelihood and those from delivered maps, which can affect $\tau$ at the $1 \sigma$ level. The spread in $\tau$ values over the ensemble of data combinations we study suggests that systematic uncertainties still contribute significantly to the current uncertainty in $\tau$, but all values are consistent with the range of $\tau=0.07 \pm 0.02$.
\end{abstract}

Keywords: cosmic background radiation - cosmological parameters - early universe

\section{INTRODUCTION}

The optical depth due to reionization, $\tau$, is one of the six defining parameters of the standard $\Lambda$ CDM cosmological model. The value of $\tau$ provides a measure of the line-of-sight free electron opacity to $\mathrm{CMB}$ radiation, and is relevant to how and when the first stars and galaxies formed. Additionally, $\tau$ shares mutual degeneracies with other $\Lambda$ CDM parameters (Zaldarriaga et al. 1997), and smaller $\tau$ uncertainties translate to more tightly constrained determinations of the curvature power $A_{s}$, the amplitude of linear matter fluctuations $\sigma_{8}$, and indirectly the Hubble constant $H_{0}$. Uncertainty in $\tau$ will limit cosmological detection of neutrino mass unless current measurements can be significantly improved upon (e.g. Allison et al. 2015; Calabrese et al. 2017; Boyle \& Komatsu 2018; Watts et al. 2018).

Although substantial progress has been made in the last few years, $\tau$ remains the least well-determined of the six $\Lambda$ CDM parameters. Lower limits on $\tau$ may be derived from the lack of the Gunn-Peterson trough (Gunn \& Peterson 1965) in the spectra of distant quasars and galaxies at redshifts $\mathrm{z} \approx 6$ (e.g. Pryke et al. 2002; Fan et al. 2006; Planck Collaboration Int. XLVI 2016). However, CMB data still provide the best means of measuring $\tau$. Specifically, the reionization bump located at power spectrum multipole moments $l<15$ in the E-mode of the $\mathrm{CMB}$ linear polarization is the most direct means for a detection, although this measurement is observation-

jweilan2@jhu.edu ally challenging. Cosmic variance places a lower limit on uncertainties that may be quoted for these low- $l$ polarization determinations: $\sigma(\tau) / \tau=2.5 \%$ for a full sky analysis, or roughly $\sigma(\tau)=0.002$ (Calabrese et al. 2017; Watts et al. 2018). The cosmic variance limit has yet to be achieved. Low multipole polarization observations are often combined with temperature and other datasets in a full likelihood to maximize constraining power on parameters (e.g. Hinshaw et al. 2013; Planck Collaboration XI 2016).

In Table 1, we show a sample of the range of determinations of $\tau$ over the last few years, as derived from WMAP and Planck CMB observations, using a variety of data combinations, analyzed sky regions, and foreground removal options. This is not a comprehensive list, but it does encompass a representative range in values. We will refer to some of the table entries throughout this discussion.

Uncertainties for $\tau$ quoted in the table represent statistical uncertainties incorporating instrumental noise and modeled CMB signal covariance; instrumental noise is the dominant contributor. In the case of $\tau$ values determined from polarization data, however, eliminating or at least characterizing potential systematic bias is an equally critical issue, which the statistical errors do not reflect. Potential sources of bias include:

- Foreground removal systematics. Polarized Galactic foreground emission outshines CMB polarization over the entire frequency range used for $\mathrm{CMB}$ observations. Foreground analyses to date rely on 
Table 1

Recently published values for $\tau$

\begin{tabular}{|c|c|c|}
\hline CMB Data & $\tau$ & Reference \\
\hline$W M A P \mathrm{TT}+\mathrm{poln}+\mathrm{BAO}+\mathrm{eCMB}^{\mathrm{a}}$ & $0.089 \pm 0.014$ & Bennett et al. (2013) \\
\hline Planck TT + LFI poln ${ }^{\mathrm{b}}$ & $0.078 \pm 0.019$ & Planck Collaboration XIII (2016) \\
\hline$W M A P+$ LFI low- $l^{\mathrm{c}}$ & $0.071 \pm 0.012$ & Planck Collaboration XI (2016) \\
\hline WMAP low- $l^{\mathrm{d}}$ & $0.067 \pm 0.013$ & Planck Collaboration XI (2016) \\
\hline Planck LFI poln ${ }^{\mathrm{e}}$ & $0.067 \pm 0.023$ & Planck Collaboration XI (2016) \\
\hline Planck TT+lens+BAO ${ }^{\mathrm{f}}$ & $0.067 \pm 0.016$ & Planck Collaboration Int. XLVI (2016) \\
\hline WMAP TT+lens+BAOg & $0.066 \pm 0.020$ & this work, Section 5 \\
\hline Planck HFI 100x143 GHzh & $0.058 \pm 0.012$ & Planck Collaboration Int. XLVII (2016) \\
\hline Planck HFI 100x143 GHz ${ }^{\text {h }}$ & $0.055 \pm 0.009$ & Planck Collaboration Int. XLVI (2016) \\
\hline
\end{tabular}

a WMAP TT+poln+BAO+eCMB = full WMAP likelihood including Baryon Acoustic Oscillations (BAO) and SPT and ACT data

b Planck TT + LFI poln = temperature and low multipole polarization likelihood

c $W M A P+$ LFI low- $l=$ low multipole WMAP and Planck likelihood, includes TE with polarization

d WMAP low- $l=$ Planck reanalysis of low multipole WMAP likelihood, includes TE with polarization

e Planck LFI poln = low multipole polarization likelihood only

${ }^{f}$ Planck TT+lens+BAO $=$ Planck temperature and weak lensing combined with BAO

g WMAP TT+lens+BAO = WMAP 9-year temperature and Planck weak lensing combined with recent $\mathrm{BAO}$

h Planck HFI 100x143 GHz = low multipole EE polarization data analysis using cross spectra from reprocessed 100 and $143 \mathrm{GHz}$ maps, with two slightly different algorithms.

observational templates to describe the spatial behavior of the foreground emission, and assume a spatially uniform scaling with frequency. Even modest errors in the template and/or its scaling with frequency can bias the derived value of $\tau$. For example, Planck Collaboration XI (2016) note that the 2013 WMAP result is roughly $1 \sigma$ lower if the Planck HFI $353 \mathrm{GHz}$ polarization map is used as a dust emission template in place of WMAP's template based on the FDS dust intensity model (Finkbeiner et al. 1999) and starlight polarization data.

- Choice of analysis mask. The choice of sky region to be analyzed is closely related to the accuracy of foreground removal: an analysis mask is used to exclude regions where foreground residuals or data systematics are believed to exceed some acceptable threshold. A commonly used mask construction method employs a threshold cut in polarized foreground intensity $P$; foreground template coefficients are often, but not always, evaluated using the same mask. Possible sources of bias are similar to those described above related to foreground removal. Potential also exists for the introduction of more subtle forms of bias; we briefly discuss one possibility in Section 3.

- Instrumental systematics. If not removed, instrumental systematics may introduce spurious sky signatures that may be mistaken for cosmological signal. For example, the 2015 Planck LFI and HFI polarization data contain known instrumental systematics that are coherent across large angular scales and produce signal artifacts at low multipoles. These systematics include leakage of temperature signal into the polarization channels (including the so-called "bandpass mismatch" correction, or BPM) and nonlinearities in the analog-to-digital converters (ADCs) in the bolometer readouts that increase effective noise levels and introduce spurious signatures (Planck Collaboration Int. XLVI 2016). Estimated BPM corrections are available for LFI frequencies and HFI $353 \mathrm{GHz}$, and have been applied to some of the 2015 data products, however the corrections are a major source of uncertainty in the polarization maps (Planck Collaboration II 2016; Planck Collaboration VIII 2016). These calibration issues rendered the 2015 HFI polarization maps unsuitable for direct cosmological studies (Planck Collaboration VIII 2016), and restricted the 2015 LFI low multipole cosmological analysis to $70 \mathrm{GHz}$ maps, after omitting survey data that failed null tests (Planck Collaboration XI 2016).

The latest Planck 2016 HFI $\tau$ determinations (Planck Collaboration Int. XLVI 2016; Planck Collaboration Int. XLVII 2016), based on reprocessed data with improved calibration, represent the tightest constraints on $\tau$ yet. Planck Collaboration Int. XLVI (2016) document the results of extensive efforts toward characterization and minimization of HFI instrumental systematics. In light of the challenging systematics removal issue, however, an independent determination of $\tau$ is prudent. The reprocessed HFI data are not yet public, so we looked at other datasets. Determinations of $\tau$ that avoid the use of large angular scale polarization data may be made if the partial degeneracy between $A_{s}$ and $\tau$ present in the temperature (TT) power spectrum is broken using CMB lensing or other large-scale structure (LSS) information, which is sensitive to the amplitude of density fluctuations but not whether photons scattered since recombination. In Table 1, we show results from two such polarizationfree determinations, which combine TT, Planck CMB lensing and BAO data. However, uncertainties are not yet competitive with the lower statistical uncertainties quoted for the HFI results. 
In this paper, we focus on determinations of $\tau$ from the WMAP and 2015 LFI polarization data. As seen in the Table 1 entry, a combined WMAP+LFI dataset can be statistically competitive with the 2016 HFI results. As well as providing data independent of HFI, WMAP and LFI sample a different frequency range than HFI and test the robustness of the result to foreground removal. The Planck Collaboration XI (2016) WMAP+LFI analysis produces a value for $\tau$ that is roughly $1 \sigma$ larger than the HFI result, and we wish to test if this result is stable when subjected to alternative analysis choices. As part of a larger effort to understand uncertainties in $\tau$, we examine shifts in $\tau$ related to different choices of foreground removal templates applied to WMAP and LFI data. We describe our method of analysis in section 2, and present individual Planck LFI and WMAP results in section 3 . In section 4 we discuss template systematics that can lead to severely compromised values for $\tau$ when analyzing WMAP and LFI data together. We discuss our conclusions and implications for $\tau$ in section 5 .

\section{DATA AND METHODOLOGY}

We use publicly available CMB polarization maps and noise covariance matrices in two Stokes parameters, Q and U, from the 2015 Planck release (Planck Collaboration I 2016) and the WMAP 9 year data release (Bennett et al. 2013) in HEALPix format (Gorski et al. 2005). From Planck, we use the 30, 70, and $353 \mathrm{GHz}$ maps and from WMAP, we use maps from the $\mathrm{K}(23 \mathrm{GHz}), \mathrm{Ka}$ $(33 \mathrm{GHz}), \mathrm{Q}(41 \mathrm{GHz})$, and $\mathrm{V}(61 \mathrm{GHz})$ bands.

Our approach is similar to the exact pixel-based likelihood methods adopted by both the Planck collaboration and the WMAP team for low- $l$ analysis (Page et al. 2007; Planck Collaboration XI 2016). For analysis that uses only polarization data, we introduce a Gaussian prior on the combination $A_{s} e^{-2 \tau}$ and fix values for other cosmological parameters from the intensity only results in Planck Collaboration XIII (2016).

The statistical properties of the CMB signal may be described as a Gaussian distributed random field on the sky using a signal covariance matrix in pixel space, $S$, computed from its power spectra, $C_{l}^{X X}$ (Tegmark \& de Oliveira-Costa 2001). If the data are a simple sum of CMB signal and Gaussian distributed instrumental noise characterized by a noise covariance matrix, $N$, the CMB likelihood function for this cleaned data, $\vec{m}=[Q, U]$, may be expressed as

$$
\mathcal{L}\left(C_{l}\right)=\frac{1}{(2 \pi)^{n_{p}}|M|^{1 / 2}} \exp \left(-\frac{1}{2} \vec{m}^{T} M^{-1} \vec{m}\right)
$$

where $M=S+N$ is the full covariance matrix of the cleaned data and $n_{p}$ is the number of pixels in each of the masked Q and U maps (Page et al. 2007). We use an MCMC sampler (emcee, Foreman-Mackey et al. 2013) to maximize the likelihood function and fit for parameters of interest and their uncertainties.

This likelihood is complicated by the need to account for foreground contamination in the raw data. In polarization, synchrotron and thermal dust are expected to be the dominant foregrounds at low and high frequencies, respectively. Taking advantage of the frequency dependence of the polarized foregrounds and CMB, a low frequency measurement may be adopted as a template for the synchrotron foreground, $\vec{T}_{s}$, and a high frequency measurement as a template for the dust, $\vec{T}_{d}$, to clean intermediate frequency bands where the CMB signal is relatively strong. We define the cleaned target map, $\vec{m}$ as

$$
\vec{m}=\vec{m}_{\nu}-\alpha_{s} \vec{T}_{s}-\alpha_{d} \vec{T}_{d}
$$

where $m_{\nu}$ are the observed $[Q, U]$ maps at frequency $\nu$, $\alpha_{s}$ scales the synchrotron template, and $\alpha_{d}$ scales the dust template. Cleaning is performed on maps in thermodynamic temperature units, and no spectral dependence constraint is placed on the template scaling parameters $\alpha_{s}$ and $\alpha_{d}$.

The templates themselves contain CMB signal that must be accounted for. The corresponding covariance matrix is given by

$$
M=\left(1-\alpha_{s}-\alpha_{d}\right)^{2} S+N_{\nu}+\alpha_{s}^{2} N_{s}+\alpha_{d}^{2} N_{d}
$$

where $N_{\nu}, N_{s}$ and $N_{d}$ are the noise covariance matrices for the frequency band of interest, the synchrotron template and the dust emission template. The equation holds for a single frequency map containing polarized CMB signal. When working with the three WMAP bands, the target map is taken as the sum of the three bands, and the covariance matrix $N_{\nu}$ is likewise the sum of their covariance matrices, whereas the signal covariance matrix is then multiplied by $\left(3-\alpha_{s}-\alpha_{d}\right)^{2}$.

In the exact case, inversion of the covariance matrix $M$ is required with each MCMC step, and the matrix dimensions corresponding to HEALPix $N_{\text {side }}=16^{1}$ resolution can inhibit computational performance. The Planck 2015 low- $l N_{\text {side }}=16$ likelihood adopted approximations that avoid repeated inversion, including pre-fitting the template coefficients, ignoring template covariance, and fixing the signal covariance to a fiducial cosmology.

We have implemented likelihood codes for use with either $N_{\text {side }}=16$ resolution maps (as with the Planck Collaboration's analysis) or $N_{\text {side }}=8$ maps (as with the WMAP team's analysis). As the cosmological EE signal is strongest for $l<15$, both resolutions are acceptable for determinations of $\tau(l<48$ vs. $l<24)$. Tests using LFI data produced similar results regardless of which code was used. Our quoted results are from our $N_{\text {side }}=8$ code implementations, as it offered greater speed of execution while recomputing and inverting the covariance matrix $M$ at every chain point. We also included options for solving for the template foreground parameters either beforehand as a separate step ("fixed" option), or simultaneously with the cosmological parameters ("free"). A simultaneous fit for all parameters of interest avoids making an intermediate approximation about the foreground cleaned noise covariance matrix and the choice of a fiducial cosmology.

Low resolution $\left(N_{\text {side }}=16\right)$ LFI Stokes Q and U maps, along with the associated covariance matrices, bandpass mismatch corrections and analysis mask, are available from the Planck Legacy Archive (PLA)2 . The LFI analysis excludes surveys 2 and 4 from use because of null-test

\footnotetext{
1 HEALPix maps are divided into $12 N_{\text {side }}^{2}$ pixels, with each pixel width $\theta_{\text {pix }} \sim 58.6^{\circ} / N_{\text {side }} . N_{\text {side }}=16$ corresponds to full-sky map of 3072 pixels. See http://healpix. sourceforge.net.

2 https://wiki.cosmos.esa.int/planckpla2015/index.php/ Map-making_LFI \\#Noise_covariance_matrices
} 
failures and we follow this recommendation. Similarly, we obtain the 9-year coadded WMAP low-resolution $\left(N_{\text {side }}=16\right)$ Stokes $\mathrm{Q}$ and $\mathrm{U}$ maps and inverse covariance matrices from $\mathrm{LAMBDA}^{3}$. The HFI $353 \mathrm{GHz}$ full mission $\mathrm{Q}$ and $\mathrm{U}$ maps are available from the PLA at high resolution $\left(N_{\text {side }}=2048\right)$ and must be degraded to low resolution. We smoothed the $353 \mathrm{GHz}$ maps to $2^{\circ}$ full width half maximum (FWHM) resolution prior to downgrading. In addition, we reconstructed the lowresolution $\left(N_{\text {side }}=16\right)$ dust template used in the LFI analysis from the Planck likelihood code (plc). As described in the Appendix, the dust template we reconstruct from the plc has large angular scale differences compared to the dust template we produce from downgrading the $N_{\text {side }}=2048 \mathrm{HFI}$ product. We further downgrade the $N_{\text {side }}=16$ products to $N_{\text {side }}=8$ using inverse noise weighting, with the exception of the smoothed high resolution $353 \mathrm{GHz}$ polarization maps, for which we use flat weighting.

\section{MISSION-SEPARATED FITS FOR $\tau$}

Before combining LFI and WMAP datasets, we analyze the WMAP and Planck LFI data separately. Either the LFI $70 \mathrm{GHz}$ Stokes Q and U maps or the combined WMAP Ka, Q and V band polarization maps provide the $\mathrm{CMB}$ signal for cosmological parameter fitting. We further adopt the Planck $353 \mathrm{GHz}$ map as the template for dust removal, but as described in Section 2 and the Appendix, we examine two choices: the "plc" version, which is extracted from the Planck low- $l$ likelihood code, and the "default" version, which we degrade from $N_{\text {side }}=2048$ to lower resolution. Consistent with the original LFI and WMAP analysis choices, we use WMAP $\mathrm{K}$-band as the template for removing synchrotron foreground from the WMAP data, and LFI $30 \mathrm{GHz}$ as the equivalent template for use in removing synchrotron from LFI $70 \mathrm{GHz}$.

We adopt the same data analysis masks as those chosen by Planck (Planck Collaboration XI 2016) and WMAP (Page et al. 2007) for low- $l$ polarization analysis. These are respectively the $\mathrm{R} 1.50$ mask, which admits roughly $46 \%$ of the sky, and the P06 mask, which admits about $75 \%$. Both were selected from a suite of masks corresponding to a series of polarized foreground intensity thresholding cuts that avoid regions of bright Galactic emission, but the details of the exact mask selection algorithm are different between the two missions. The WMAP diffuse P06 mask was chosen, "as the best compromise between maximizing usable sky area while minimizing foreground contamination" (Page et al. 2007). Planck Collaboration XI (2016) select an optimum mask based on $\chi^{2}$ minimization of foreground templates fit to the observed LFI $70 \mathrm{GHz}$ data as a function of successively increasing sky area. Use of this technique comes with caveats not discussed in Planck Collaboration XI (2016). Minimizing the variance in the template-cleaned map acts to maximize chance correlations between the CMB signal and e.g. foreground templates, noise and systematics. These chance alignments remove power from the cleaned map, which will bias $\tau$ to lower values.

\footnotetext{
3 https://lambda.gsfc.nasa.gov/product/map/dr5/ m_products.cfm
}

The degree of bias resulting from this effect is dependent on a number of factors, including masked sky fraction and spatial morphology of the foregrounds. In the case where foreground templates perfectly match the observed foregrounds, simulations indicate that power in a R1.50 masked cleaned map can be biased low by at much as $5-10 \%$ through chance foreground alignments. However, chance alignments are not the only sources of error in the cleaned map. Use of imperfect foreground templates in the map-cleaning process and/or the presence of remnant systematic signatures produces non-cosmological residuals that can produce a positive $\tau$ bias. The $\chi^{2}$ process minimizes the combination of correlation bias (negative) and template errors (generally positive). The situation is thus complex and requires an understanding of an experiment's dominant sources of uncertainty. For future analyses, we suggest a study of mask-related uncertainties based on simulations.

Table 2 shows a comparison between our results for $\tau$ (row 2) derived under the same template and masking conditions as those utilized in the Planck likelihood (row 1). WMAP results for the same template and masking conditions are similar (row 4). However, use of the "default" dust template, a different analyzed sky fraction, and/or foreground coefficients determined using a different sky fraction can produce up to $\sim 1 \sigma$ shifts in these values (rows $3,5,6$ ).

Some of these shifts may simply be statistical, while others may be from introduced bias. The table illustrates the need for an organized and systematic examination of results with regard to masking and template choices, within the context of the same cosmological dataset. We proceed toward using $W M A P+\mathrm{LFI}$ for that purpose.

\section{AN UNADDRESSED SYSTEMATIC}

As template choices for removing synchrotron, WMAP K-band and LFI $30 \mathrm{GHz}$ polarization maps should be almost interchangeable, given both the similar signal-tonoise (Planck Collaboration XXV 2016) and their relative proximity in frequency. In a combined $W M A P+\mathrm{LFI}$ analysis, it would simplify the computation to use only one synchrotron template, and bolster confidence in the quality of Planck BPM correction if results using K-band were the same as those using the LFI $30 \mathrm{GHz}$ data.

We next perform foreground cleaning of WMAP KaQV data using the Planck $30 \mathrm{GHz}$ synchrotron template, and conversely, clean LFI $70 \mathrm{GHz}$ using the WMAP K-band synchrotron template, holding all other analysis assumptions constant. The last two rows of Table 2 show the resultant values for $\tau$ when using these alternate choices of the synchrotron template. In both cases, $\chi^{2}$ values are significantly increased, as are the derived $\tau$ values. The $70 \mathrm{GHz}$ result returns $\tau=0.094 \pm 0.016$, in tension with the HFI results listed in Table 1. WMAP KaQV cleaned using the LFI $30 \mathrm{GHz}$ synchrotron template returned $\tau$ near 0.37 , a result that may be rejected as unrealistic based on existing CMB temperature observations (see e.g. the TT+lens+BAO entries in Table 1). At best, neither result argues for a well-understood and consistent representation of the polarized synchrotron template, so we needed to understand these results before proceeding further.

A solution to this problem lies with a large angular scale residual signature in $\mathrm{Q}$ and $\mathrm{U}$ that becomes appar- 
Table 2

Derived values for $\tau$, EE only

\begin{tabular}{|c|c|c|c|c|c|c|c|c|}
\hline Row & CMB Data & $\begin{array}{l}\text { Synchrotron } \\
\text { Template }\end{array}$ & $\begin{array}{l}\text { Dust } \\
\text { Template }^{a}\end{array}$ & fg coeffs ${ }^{b}$ & $f_{\text {sky }}{ }^{\mathrm{c}}$ & $\tau$ & $\chi_{\text {maxlike }}^{2} /$ dof & Reference \\
\hline 1 & LFI $70 \mathrm{GHz}$ & LFI $30 \mathrm{GHz}$ & plc & fixed & $46 \%$ & $0.052 \pm 0.023$ & - & Planck $^{\mathrm{e}}$ \\
\hline 2 & LFI $70 \mathrm{GHz}$ & LFI $30 \mathrm{GHz}$ & $\mathrm{plc}$ & fixed & $46 \%$ & $0.053 \pm 0.023$ & $728 / 734$ & this work \\
\hline 3 & LFI $70 \mathrm{GHz}$ & LFI $30 \mathrm{GHz}$ & default & free & $46 \%$ & $0.076 \pm 0.020$ & $823 / 734$ & this work \\
\hline 4 & WMAP KaQV & $W M A P \mathrm{~K}$ & plc & free & $46 \%$ & $0.045 \pm 0.019$ & $754 / 734$ & this work \\
\hline 5 & WMAP KaQV & WMAP K & plc & fixed $^{\mathrm{d}}$ & $46 \%$ & $0.040 \pm 0.017$ & $752 / 734$ & this work \\
\hline 6 & WMAP KaQV & WMAP K & default & fixed $^{\mathrm{d}}$ & $75 \%$ & $0.058 \pm 0.017$ & $1190 / 1162$ & this work \\
\hline 7 & LFI $70 \mathrm{GHz}$ & WMAP K & $\mathrm{plc}$ & free & $46 \%$ & $0.094 \pm 0.016$ & $846 / 734$ & this work \\
\hline 8 & WMAP KaQV & LFI $30 \mathrm{GHz}$ & $\mathrm{plc}$ & free & $46 \%$ & $0.37 \pm 0.02$ & $933 / 734$ & this work \\
\hline
\end{tabular}

a "plc" refers to the low resolution $353 \mathrm{GHz}$ dust template reconstructed from the Planck likelihood code; "default" refers to the low resolution $353 \mathrm{GHz}$ dust template degraded from the delivered high resolution maps.

b Foreground template coefficients are either free parameters in the likelihood, or fit in a separate step beforehand and are fixed parameters in the likelihood.

${ }^{c}$ Fraction of sky used in analysis, corresponding to the Planck R1.50 analysis mask (46\%) and the WMAP P06 analysis mask (75\%)

${ }^{\mathrm{d}}$ Foreground template coefficients were computed using a larger sky region than that used for analysis.

e Planck Collaboration XIII (2016)
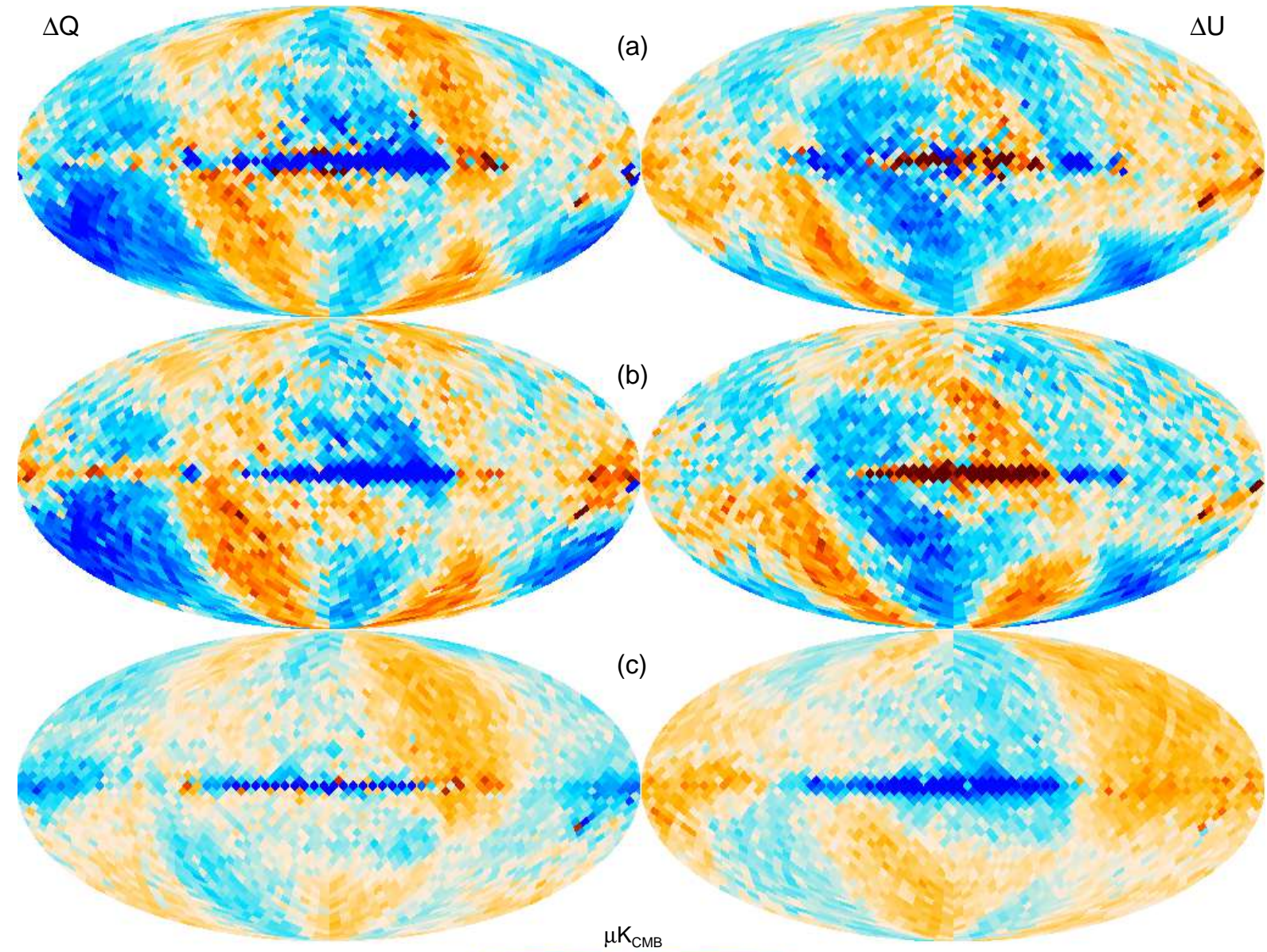

(c)

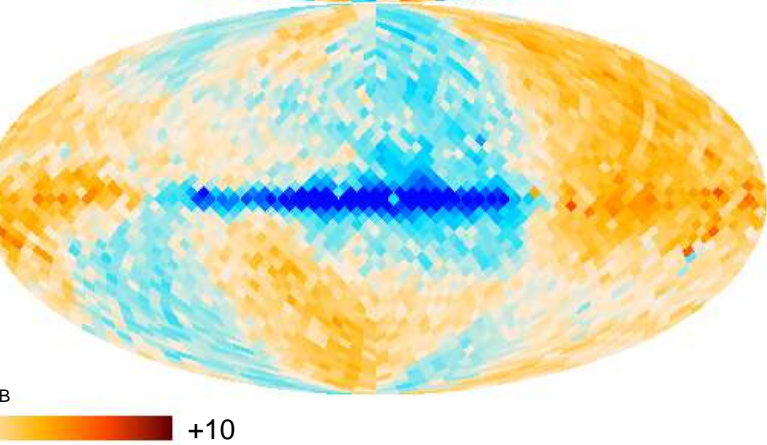

Figure 1. Differences between independent Planck and WMAP Stokes Q and U maps at similar frequencies reveal a large angular scale signature at high Galactic latitudes that does not correspond to any sky signal morphology (this statement ignores the Galactic plane differences running horizontally across the center of each map). Such a $\Delta Q, \Delta U$ signature has potential to influence the quality of synchrotron foreground removal through the use of $30 \mathrm{GHz}$ and K-band templates, and thus bias the determination of $\tau$. (a) The difference between the derived Planck Commander synchrotron component at $30 \mathrm{GHz}$ (called $S_{30}$ ), and WMAP K-band scaled to that frequency (similar to Figure 46 of Planck Collaboration X 2016). (b) Bandpass mismatch corrected LFI 30 GHz minus WMAP K-band, scaled to an effective frequency of $30 \mathrm{GHz}$ using the same $\nu^{-3,2}$ dependence as in (a). Features similar to (a) persist, indicating that any Planck contribution is not solely confined to the LFI $44 \mathrm{GHz}$ band. (c) $S_{30}$ minus scaled $30 \mathrm{GHz}$, or equivalently, (a)-(b), since the WMAP K-band contribution drops out of the difference. This establishes the existence of large angular scale structure that is limited to Planck alone, and shares common morphological features with (a) and (b). 
ent only when comparing WMAP and LFI polarization maps. Planck Collaboration X (2016) note the existence of large angular scale signatures unrelated to sky signal when differencing the empirically modeled Commander synchrotron component against WMAP K-band observations. The difference between the Commander synchrotron Q, U maps at $30 \mathrm{GHz}\left(S_{30}\right)$ and WMAP $\mathrm{K}$ band $\mathrm{Q}, \mathrm{U}$ is performed after scaling the K-band maps to $30 \mathrm{GHz}$ using a $\nu^{-3.2}$ power law dependence. The scaling is chosen to null the synchrotron foreground using a representative spectral index at high Galactic latitudes. Since synchrotron is the dominant K-band foreground emission component, the difference should primarily show white noise, as the polarized CMB signal is completely subdominant to instrument noise for these maps. The $\mathrm{Q}$ and $\mathrm{U}$ components of an $S_{30}-0.39 \mathrm{~K}$ difference map are shown in the top row of Figure 1. The difference is dominated by a residual large angular scale pattern at high Galactic latitudes with a peak amplitude near $\pm 10 \mu \mathrm{K}$, far in excess of the expected cosmological signal. Planck Collaboration X (2016) note "a morphology clearly associated with the scanning strategy of either Planck or WMAP", and attribute the most likely origin of this pattern to Planck $44 \mathrm{GHz}$ (because the $44 \mathrm{GHz}$ data failed null tests at low multipoles) and to WMAP low multipole "poorly measured modes," which are modes of enhanced noise variance related to the WMAP scan strategy (Page et al. 2007; Jarosik et al. 2011; Bennett et al. 2013). In a different analysis, Planck Collaboration XXV (2016) compute the difference between the combined weighted Planck LFI and WMAP polarization $\mathrm{P}$ maps, defined as $\left[\left(Q_{\mathrm{LFI}}-Q_{W M A P}\right)^{2}+\right.$ $\left.\left(U_{\text {LFI }}-U_{W M A P}\right)^{2}\right]^{1 / 2}$. They also note large angular scale residuals at high latitudes, and attribute these primarily to WMAP poorly measured modes. Differences in the Galactic plane are assumed to arise from spectral index variations between frequencies that are not accounted for under the assumption of a fixed high Galactic latitude spectral index.

The attribution of the residual signature to WMAP poorly measured modes carries consequences only in terms of visual presentation, as it is not a true systematic residual. When constructing and viewing maps without accounting for variance, as we have in Figure 1, there can appear to be large angular scale structure because of the enhanced noise. However, within the context of a low multipole likelihood, where full covariance matrices are included, poorly measured modes are correctly weighted in the analysis (Page et al. 2007).

Isolating the root source of any residual signature that may be present in the Commander $S_{30} \mathrm{Q}, \mathrm{U}$ maps is complicated by the nature of its production, as it relies on assumed foreground modeling dependencies and is derived by fitting these models to a combined set of LFI and HFI polarization maps. Therefore, we simplify the analysis by computing the difference between two frequencies only: the full-mission LFI $30 \mathrm{GHz}$ and WMAP K-band Q, U maps, both scaled to the effective frequency of $30 \mathrm{GHz}$ using a $\nu^{-3.2}$ dependence: $\Delta[Q, U]=0.85[Q, U]_{\text {LFI } 30}-0.39[Q, U]_{\text {WMAP K }}$, shown in Figure $1 \mathrm{~b}$ at HEALPix resolution $N_{\text {side }}=16$. (All maps in Figure 1 are shown at a fiducial frequency of $30 \mathrm{GHz}$ for ease of intercomparison). Although "30-K" (Fig- ure $1 \mathrm{~b})$ is slightly noisier in appearance than " $S_{30}-\mathrm{K}$ " (Figure 1a), the spatial correspondence between highlatitude features is quite similar between the two: the linear Pearson correlation coefficient is 0.9 for Galactic latitudes $|b|>10^{\circ}$. Since the large-scale signature is identified via a difference between LFI and WMAP data, its origin may lie with either Planck or WMAP data, or both. However, Figure $1 \mathrm{~b}$ illustrates that if there is a Planck contribution to this signature, then $30 \mathrm{GHz}$ is involved, not only $44 \mathrm{GHz}$ as suggested by Planck Collaboration X (2016).

In Figure 1c, we also show the difference between $S_{30}$ and LFI $30 \mathrm{GHz}$, or equivalently between " $S_{30}-\mathrm{K}$ " and "30-K". This last figure illustrates the presence of large angular scale structure associated with Planck analysis products only, although the difference is between data and a model that fits that data. However, Planck Collaboration X (2016) illustrate examples of the model's ability to fit actual sky signal while isolating residuals associated with calibration artifacts, as in the destriper signatures in temperature data. It is likely then that the " $S_{30}-30$ " residual is associated with calibration artifacts in Planck polarization data (see also Wehus \& Eriksen 2017). This Planck-related large angular scale structure shares common morphological features with " $S_{30}-\mathrm{K}$ " and " $30-\mathrm{K}$ ", although not necessarily of the same sign. As there is no publicly available Commanderlike foreground polarization model derived using only WMAP data, an equivalent " $S_{\mathrm{K}}-\mathrm{K}$ " map is not shown. The WMAP MCMC foreground polarization models presented by Bennett et al. (2013) cannot serve as estimators of potential K-band residual structure, since certain model parameters are specifically fixed to values computed directly from K-band data. The morphology and amplitude of K-band poorly measured modes (a noise property and not a systematic error) are discussed in the next section. We note that the differences shown in Figure 1 are not particularly sensitive to the choice of spectral index for scaling maps to the fiducial frequency, that the magnitude of the residual is not consistent with spectral index variations over the sky, and that the exclusion of LFI surveys 2 and 4 does not appreciably alter the morphology shown.

\subsection{Morphological template fits}

We attempted to determine if plausible mechanisms associated with calibration uncertainties and/or noise properties could produce the morphology of the "30 K" residuals shown in Figure $1 b$. Specifically, we constructed Q, U template maps whose origin could be associated with a physically-motivated mechanism arising either solely from Planck LFI $30 \mathrm{GHz}$, or solely from WMAP K-band. For Planck LFI, the documented source of largest uncertainty lies with the correction for leakage of intensity into polarization (Planck Collaboration II 2016). We constructed a set of templates to reflect possible differences between the "true" leakage correction and that actually applied. We used the $30 \mathrm{GHz}$ full mission temperature map and transformed it to $\mathrm{Q}$, $\mathrm{U}$ leakage maps via cosine and sine of the geometrical angle computed from the IQ and IU full mission covariance maps, and also performed a similar computation of far-sidelobe leakage templates using the temperature maps from Figure 24 of Planck Collaboration III (2014). 


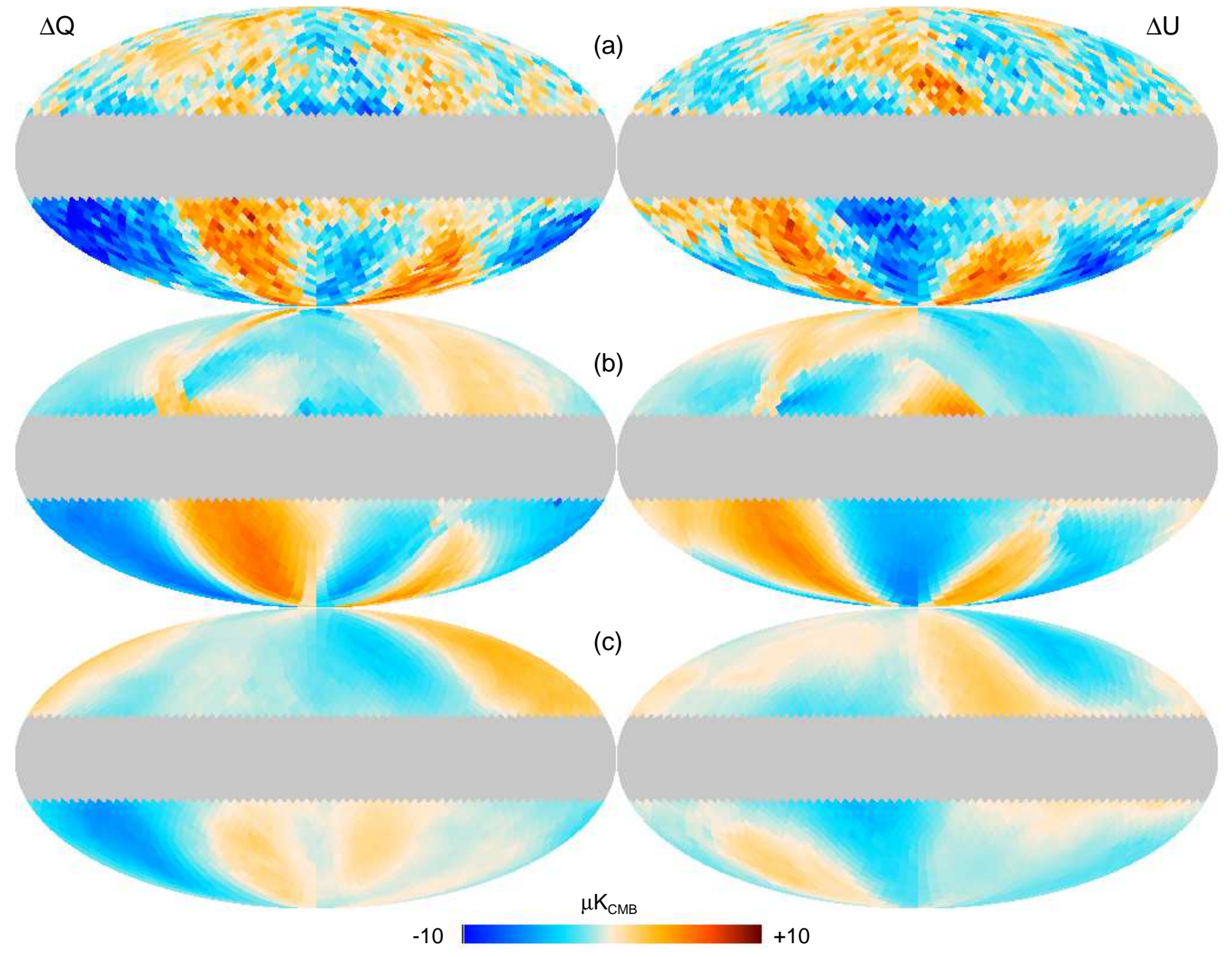

Figure 2. Based on morphological arguments, we conclude that Planck contributions to the large-scale $[\Delta \mathrm{Q}, \Delta \mathrm{U}]$ signature in Figure 1 cannot be discounted and are likely the dominant source. (a) Same as Figure 1b, i.e. polarized LFI 30 GHz minus WMAP K scaled to $\nu_{\text {eff }}=30 \mathrm{GHz}$. We fit combinations of likely noise and calibration-related templates to this signature (see text), and present fits that attribute the morphology either wholly to Planck-related causes or wholly to WMAP-related causes. The grey band is a $\pm 20^{\circ}$ cut about the Galactic plane, defining the fit mask. (b) An empirical fit to the $\Delta \mathrm{Q}$ and $\Delta \mathrm{U}$ pattern in (a) using templates that assume the structure is attributable to leakage of Planck intensity into Q and U (see text). (c) As with (b), but using templates characterizing WMAP poorly measured modes on large angular scales and loss imbalance uncertainty.

The templates are arbitrarily normalized since they are designed to approximate morphology only and do not reflect leakage efficiency. The delivered $30 \mathrm{GHz}$ BPM full mission Q, U correction maps were also included in the LFI template set. For WMAP K-band, we include Q, U templates for the poorly measured modes, and the two forms of loss imbalance correction maps (Jarosik et al. 2007) included in the WMAP final product delivery. A consideration in choosing templates was to limit the basis set to the most likely candidates, and include nothing further, since a sufficiently large number of templates and degrees of freedom can reproduce almost any morphology without regard to actual physical cause. We empirically fit the large angular scale high latitude "30 $\mathrm{K}$ " $\Delta \mathrm{Q}, \Delta \mathrm{U}$ signatures using an unweighted linear combination of spatial templates described above. Fits were performed to $\Delta \mathrm{Q}, \Delta \mathrm{U}$ data outside of $|b| \geq 20^{\circ}$, in order to exclude Galactic plane regions most affected by spectral index variations and BPM correction uncertainties, where the template would be least accurate. $\Delta \mathrm{Q}$ and $\triangle \mathrm{U}$ were fit separately to the WMAP and LFI template basis sets. Figure $2 \mathrm{~b}$ shows the resulting fit using the LFI template basis set, while Figure $2 \mathrm{c}$ shows that using the WMAP template basis set. These may be compared visually against the input systematic maps in the top row of the figure. Neither of the combinations exactly reproduce the input $[\Delta \mathrm{Q}, \Delta \mathrm{U}]$ signature: the linear correlation coefficient between $[\Delta \mathrm{Q}, \Delta \mathrm{U}]$ and the two empirical fits is 0.76 for the LFI basis set, and 0.46 for the WMAP basis set. The somewhat stronger morphological agreement between the input signature and the LFI fit, coupled with the apparent presence of large angular scale structure isolated to Planck alone, leads us to suspect a dominant contribution from the LFI $30 \mathrm{GHz}$ map.

Amplitude arguments also favor a stronger association with the LFI $30 \mathrm{GHz}$ map. When the empirical templates in Figure 2b and c are peak-normalized and used as basis functions in a linear fit to the "30 - K" $\Delta \mathrm{Q}$, $\Delta \mathrm{U}$ signatures, we derive an LFI template coefficient of $5 \mu \mathrm{K}$, but only $0.7 \mu \mathrm{K}$ for the WMAP template. The WMAP template coefficient is within expectations for poorly measured modes described by the K-band covariance matrix and extrapolated to $30 \mathrm{GHz}$.

However, if the signature is limited to the LFI $30 \mathrm{GHz}$ band, we would expect greater disagreement between values for $\tau$ derived from the WMAP-only and LFI-only 
$\mathrm{f}$

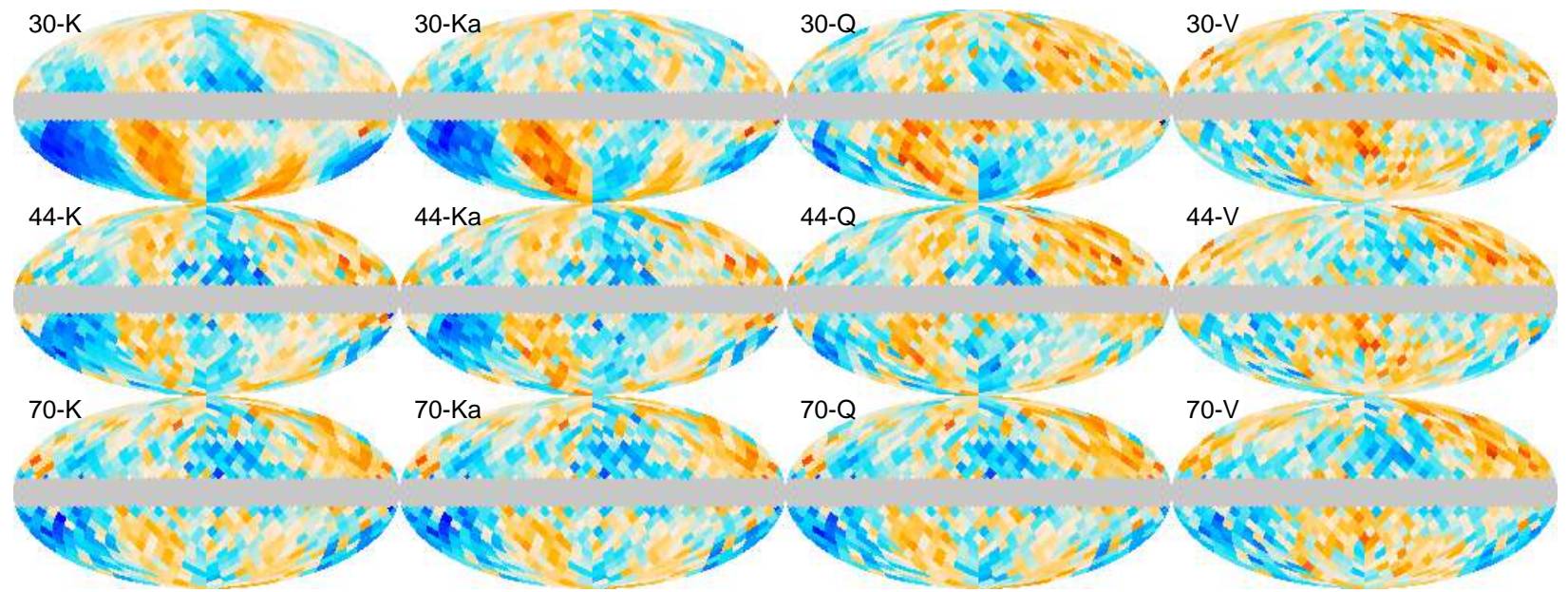

Figure 3. Stokes Q foreground-nulled band-pair maps for WMAP-LFI frequency pairs. The grey band excludes Galactic latitudes $|b|<10^{\circ}$. Synchrotron foreground has been nulled using a $\nu^{-3.2}$ spectral dependence, and dust emission removed from V-band and $70 \mathrm{GHz}$ using the Planck Commander foreground model. The resultant residuals reflect instrument noise, cosmological signal and imperfections in foreground removal that are both primarily subdominant to the noise, and a large-scale signature with strong features of order $5-10 \mu \mathrm{K}$ in the 30-K combination. Each band-pair image is autoscaled using an outlier-resistant determination of extrema, so that the morphology in each image is readily apparent. The residual large-scale signature is most clearly visible in the $30-\mathrm{K}$ combination, but visually persists in other frequency combinations. Taken in context with Figure 4, we infer a common-mode systematic across frequencies.

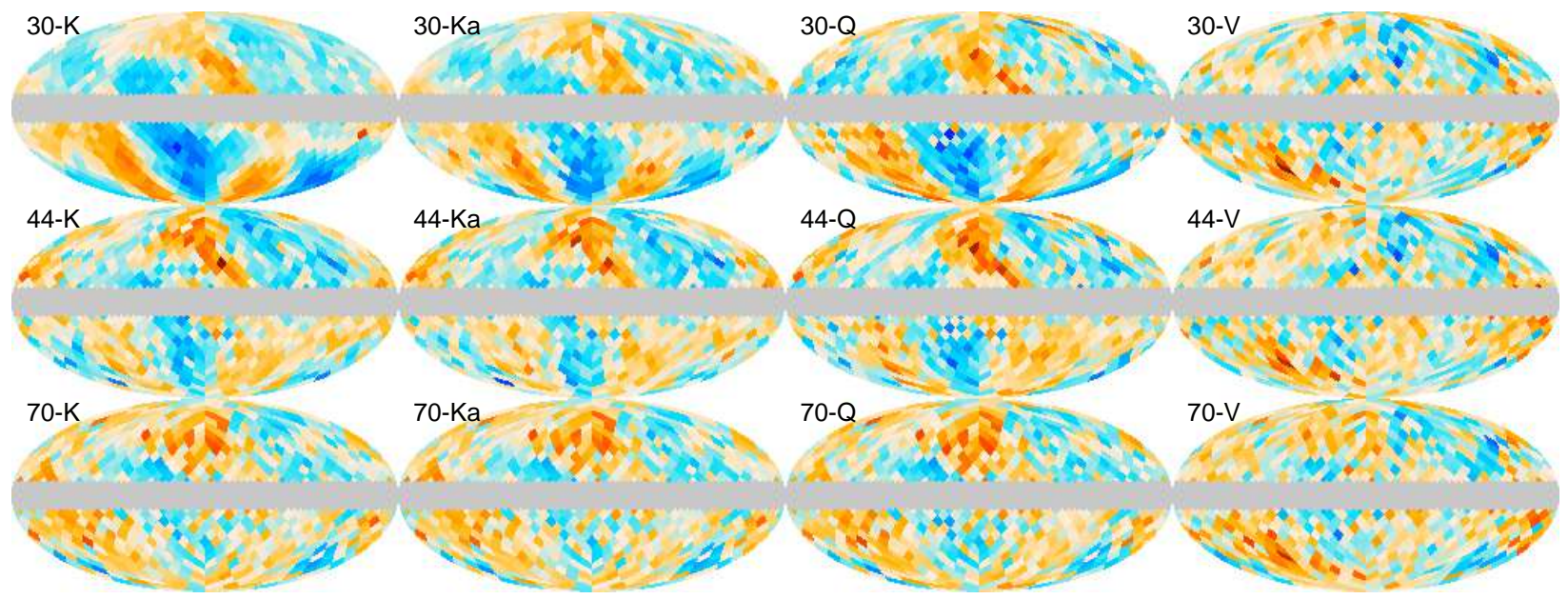

Figure 4. Same as for Figure 3, but for the corresponding Stokes U nulled band-pair maps for WMAP-LFI pairs. See Figure 3 caption for further explanation.

fits in rows 2 and 4 of Table 2 . A $5-10 \mu \mathrm{K}$ systematic in the $30 \mathrm{GHz}$ synchrotron template would scale down to roughly a $0.3-0.7 \mu \mathrm{K}$ systematic at $70 \mathrm{GHz}$, which is sufficiently large compared to the predicted polarized CMB signal to cause noticeable bias in recovered values of $\tau$.

\subsection{Spectral behavior}

We investigated to what extent the $[\Delta \mathrm{Q}, \Delta \mathrm{U}]$ signature could be detected in WMAP and LFI frequency combinations other than $30 \mathrm{GHz}$ and $\mathrm{K}$-band. We examined both WMAP-only and LFI-only combinations as well as WMAP-LFI combinations involving WMAP K, Ka, Q and V bands, and the LFI 30, 44 and $70 \mathrm{GHz}$ bands. We formed "band-pair" maps from the weighted linear combination of two independent frequencies. Weights for each frequency were computed to null a $\nu^{-3.2}$ brightness temperature spectrum, yet preserve the underlying
CMB signal, such that $\mathrm{wt}\left(\nu_{1}\right)+\operatorname{wt}\left(\nu_{2}\right)=1$ when applied to maps in thermodynamic temperature units. Because only one foreground spectral constraint can be applied with this weighting scheme, we removed the Commander polarized dust emission model from all frequencies beforehand. The dust emission removal is of minor importance for all frequencies except V-band and $70 \mathrm{GHz}$. Identical weights are applied to the $\mathrm{Q}$ and $\mathrm{U}$ maps of each band-pair. Stokes Q WMAP-LFI pair differences are shown in Figure 3, and those for Stokes U in Figure 4. Each individual band-pair image is autoscaled using an outlier-resistant minima and maxima determination scheme, so that neighboring images may be more easily compared visually despite noise variations. Features become less distinct for band-pair combinations that include at least one of the higher frequency maps, as their signal-to-noise is lower. With the exception of the $70-\mathrm{V}$ combination, combinations including 70 or $\mathrm{V}$ 


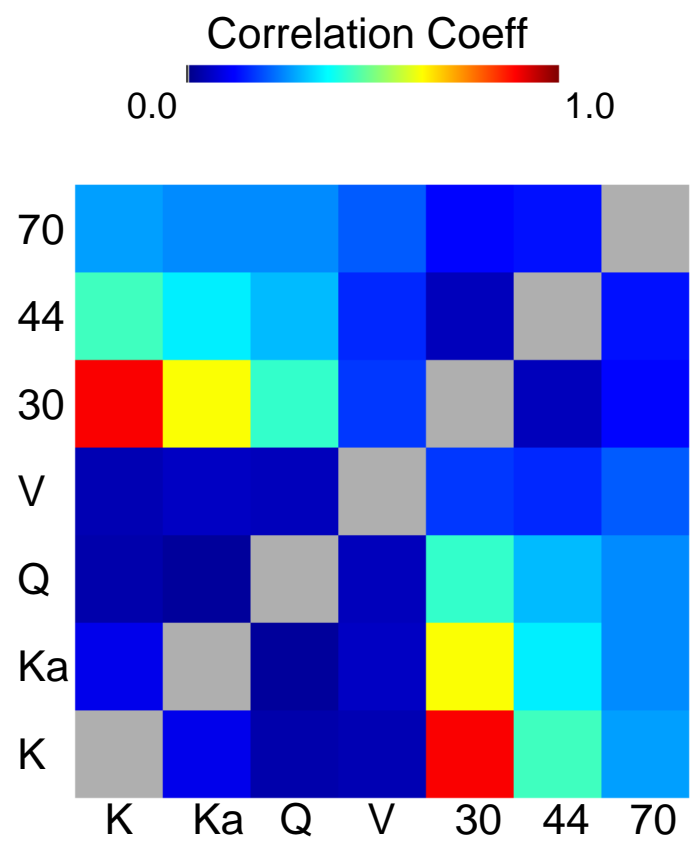

Figure 5. The absolute value of the linear correlation coefficient between the " $S_{30}-K$ " map shown in Figure $1 \mathrm{a}$ and each of the LFI and WMAP [Q, U] band-pair difference maps shown, for example, in Figures 3 and 4. By construction, the matrix is symmetric. The correlation coefficients for WMAP-LFI pair differences are shown in the upper left quadrant and duplicated in the lower right. The lower left quadrant shows the correlation for WMAP-internal combinations, and the upper right for LFI-internal combinations. Only WMAP-LFI band-pair combinations show significant correlation with the systematic. We deduce that the systematic signature has a frequency dependence that matches the nulled foreground spectrum in the band-pair maps (see text).

are heavily weighted toward these frequencies and do not change substantially in appearance as the companion frequency varies. Combinations that include WMAP Vband include the visual signature from poorly measured modes. The "30-K" signature persists in WMAP-LFI combinations involving $\mathrm{K}, \mathrm{Ka}, \mathrm{Q}, 30 \mathrm{GHz}$ and $44 \mathrm{GHz}$, with less certainty about $70 \mathrm{GHz}$ and $\mathrm{V}$. We infer a systematic error that is common-mode across frequencies.

We linearly correlated the " $S_{30}-K$ " $[\mathrm{Q}, \mathrm{U}]$ templates against each of the WMAP- and LFI-internal combinations and the WMAP-LFI pair combination $[\mathrm{Q}, \mathrm{U}]$ maps. The correlation was performed outside Galactic latitude $|b| \geq 10^{\circ}$, and treated [Q,U] simultaneously in the solution. We show the correlation coefficients as a symmetric matrix in Figure 5, and show the absolute value of the correlation coefficient, since sign is irrelevant in this context. Significant correlations are found only in the WMAP-LFI pair combinations. The "30-K" signature does not correlate strongly in the LFI-internal combinations containing $30 \mathrm{GHz}$, nor are there strong correlations in the WMAP-internal combinations that include K-band. The simplest explanation is that the signature itself possesses a frequency dependence that closely matches that of $\nu^{-3.2}$. A systematic isolated to LFI Q, $\mathrm{U}$ maps with a frequency dependence that matches the nulled foreground spectrum would be undetectable in the LFI-internal band-pair combinations, and only appear in WMAP-LFI pair combinations. Such a systematic would also evade LFI-internal null tests based on in-band jack-knife tests. The poorly measured modes associated with WMAP do not match a foreground spectrum signature, whereas such a spectrum is a natural consequence from leakage of strong Galactic temperature signal into LFI polarization maps. The WMAP sky scan pattern probes a larger range of scan angle orientations than does Planck's scan pattern, so it is reasonable to expect that the leakage would be of far greater concern for Planck than for WMAP polarization data (Planck Collaboration II 2016; Page et al. 2007).

\subsection{Effect of increased masking and multipole cuts}

We applied two common techniques that attempt to mask or otherwise mitigate the systematic signature introduced when cleaning LFI data with a WMAP synchrotron template and vice-versa. In the first method, we constructed a variety of masks seeking to define smaller areas of sky less affected by non-CMB signals. Our most restrictive mask excluded regions of the Q and U " $30-\mathrm{K}$ " signature with absolute amplitudes $>2 \mu \mathrm{K}$, in combination with the Planck R1.50 analysis mask, retaining only $11 \%$ of the sky. Chains duplicating rows 7 and 8 of Table 2 but using this new mask returned $\tau$ for WMAP cleaned with LFI $30 \mathrm{GHz}$ of $0.24 \pm 0.03$, and $0.09 \pm 0.03$ for LFI $70 \mathrm{GHz}$ cleaned with WMAP K-band. None of the masks we explored produced mean $\tau$ values similar to those found when using WMAP-internal or LFI-internal data and synchrotron template combinations. The second technique excluded data in harmonic space but retained the original R1.50 pixel analysis mask. Motivated by a statement in Planck Collaboration X (2016) that the " $\mathrm{S}_{30}-\mathrm{K}$ " signature is dominated by modes for multipoles 2 and 3, we excluded these multipoles and re-ran chains under the same foreground cleaning and masking conditions as specified in rows 7 and 8 of Table 2. This method produced $\tau$ values less than $1 \sigma$ different from those derived using the full multipole range.

\subsection{Implications for $\tau$}

What are the consequences for $\tau$ with an LFI temperature to polarization leakage that matches the sky spectrum? As long as LFI $30 \mathrm{GHz}$ is used as the synchrotron template for LFI $70 \mathrm{GHz}$, and the morphology of the systematic is frequency independent, then the recovered values for $\tau$ should be relatively unaffected if the template foreground coefficients accurately reflect the foreground contribution. The relative agreement between the WMAP-internal and LFI-internal $\tau$ results under the same masking conditions implies at least some consistency with this picture. However, we cannot quantitatively assess the degree to which these conditional statements are met, and there may be additional issues such as somewhat different spectral index dependencies between temperature and polarization. Use of LFI $30 \mathrm{GHz}$ as a template for cleaning non-LFI datasets such as HFI data would produce a biased result, assuming there is no equivalent signature in the other dataset. The 2016 HFI $\tau$ analysis (Planck Collaboration Int. XLVI 2016) makes use of LFI polarization data in several ways: as templates for residual systematic evaluation, as foreground templates $(30 \mathrm{GHz})$, and in EE cross-spectra (70x100 GHz, 70x143 GHz) for confirmation of results 
from 100x143 GHz. Synchrotron emission is not a large contributor at $100 \mathrm{GHz}$, and even less at $143 \mathrm{GHz}$, so bias introduced by use of the LFI $30 \mathrm{GHz}$ template should be low. We suggest a useful test of robustness of the HFI result would include substituting equivalent WMAP data for LFI data in the analysis.

\section{CONCLUSIONS}

The reionization optical depth, $\tau$, is currently the least well constrained of the six standard $\Lambda$ CDM parameters. Large angular scale microwave polarization data provide the means for direct measurement of $\tau$, but Galactic foreground emission must be removed with sub- $\mu \mathrm{K}$ accuracy to expose the underlying cosmological signal. Foreground removal using templates, a technique employed by both WMAP and Planck, introduces uncertainties associated with accuracy of the templates, including decoherence of the template morphology with frequency and systematics in the templates. Instrumental noise and data systematics also contribute to uncertainties, requiring some tailoring of analysis choices to individual datasets.

In this paper, we have pursued combined analysis of WMAP and Planck LFI polarization data as a means of cross-validating published $\tau$ values derived using publicly unavailable HFI 100 and $143 \mathrm{GHz}$ Stokes Q and U maps. In doing so, we have encountered a few issues:

1. Analysis mask selection techniques involving $\chi^{2}$ minimization act to maximize bias from chance correlations of CMB signal with foreground templates, noise and systematics. The importance of this bias term in the uncertainty budget depends on what additional sources of map-cleaning error are present and their relative dominance. In general, there is no substitute for carefully assessing each specific situation. We recommend a systematic approach to analysis mask selection through simulations.

2. There is an inconsistency between the $353 \mathrm{GHz} \mathrm{Q}$ and $U$ thermal dust template maps internal to the Planck low multipole likelihood code, and those available as delivered maps from the Planck Legacy Archive. The mismatch produces a $1 \sigma$ shift in $\tau$ derived from the LFI $70 \mathrm{GHz}$ data.

3. An unexpectedly high value for $\tau$ is derived when WMAP K-band Stokes Q and U maps are used to clean synchrotron emission from polarized Planck LFI $70 \mathrm{GHz}$ data, and a severely compromised $\tau$ value results when LFI $30 \mathrm{GHz} \mathrm{Q}$ and $\mathrm{U}$ maps are used to clean synchrotron from the combined WMAP Ka, Q and $\mathrm{V}$ bands.

Template systematics are the root cause of the synchrotron cleaning issue. Differences between combined LFI polarization bands and their WMAP frequency counterparts have revealed residual structure on large angular scales (Planck Collaboration X 2016; Planck Collaboration XXV 2016). The Planck Collaboration concluded that this residual pattern primarily arises from WMAP poorly measured modes, with any Planckrelated contribution limited to the LFI $44 \mathrm{GHz}$ band. We reject both of these assertions and show evidence that the signature is a systematic internal to LFI and roughly common-mode to all LFI bands. The spectral behavior of the systematic scales like the intensity signal and is thus likely to arise from temperature-to-polarization leakage in the LFI channels. In an LFI-only analysis this systematic is largely nulled by the foreground removal procedure, but attempting to combine WMAP with Planck data before foreground removal creates an inconsistency that greatly compromises the use of both data sets together. We were not able to mitigate the effects of the systematic either through additional masking or removal of multipoles 2 and 3 .

Planck Collaboration XI (2016) derive $\tau=0.071 \pm$ 0.012 from a combined WMAP+LFI analysis (see Table 1). This result is based upon a weighted coaddition of cleaned maps consisting of WMAP data cleaned with K-band and $353 \mathrm{GHz}$ over $75 \%$ of the sky, and LFI 70 $\mathrm{GHz}$ data cleaned with LFI $30 \mathrm{GHz}$ and $353 \mathrm{GHz}$ over $46 \%$ of the sky. Although such a combination produces a result for $\tau$ that is not unreasonable, we cannot assess the associated systematic uncertainty accurately. In addition to the mix of analysis masks, the LFI temperature to polarization leakage efficiency may not be constant with frequency, and production of a correction template would be circular since at present it would be based on WMAP and LFI data. As a comparison standard for other $\tau$ determinations, we believe this particular result is compromised, but should be re-examined when an updated LFI calibration becomes available.

Unfortunately, the levels of systematics, foregrounds, and the quality of the foreground templates are not often well known. Robustness can be estimated by fitting a range of $\mathrm{CMB}$ maps with a range of templates under different masking assumptions. We note the spread in results for $\tau$ both in Table 1 and Table 2 (excluding rows 7 and 8), and comment that the quoted uncertainties for $\tau$ are most likely underestimated because systematic uncertainties are not included. However, tension is not high between the quoted values because of the relatively large uncertainties and all are consistent with a polarizationderived value of $\tau=0.07 \pm 0.02$, used as a prior in some earlier studies (Planck Collaboration XI 2016; Addison et al. 2016; Aylor et al. 2017). Values for $\tau$ derived without the use of polarization data are also consistent with this range. Planck Collaboration Int. XLVI (2016) derived $\tau=0.067 \pm 0.016$ for Planck TT+lens+BAO (see Table 1). Adopting the analysis methods and BAO dataset selection of Addison et al. (2018), we derive a similar value, $\tau=0.066 \pm 0.020$, for the combination of WMAP 9-year TT, Planck 2015 weak lensing, and BAO.

There is considerably more work to be done in terms of characterizing $\tau$ and choosing the optimal analysis masks and foreground removal given instrumental properties. Future datasets should also assist with the analysis. These include the final Planck data release, additional weak lensing constraining power from groundbased surveys such as ACTPol and SPTpol, and high signal-to-noise polarization observations from e.g. the Cosmic Large Angular Scale Survey (CLASS), which is projected to recover $\tau$ to nearly cosmic variance uncertainties and enable a $4 \sigma$ detection of the sum of the neu- 


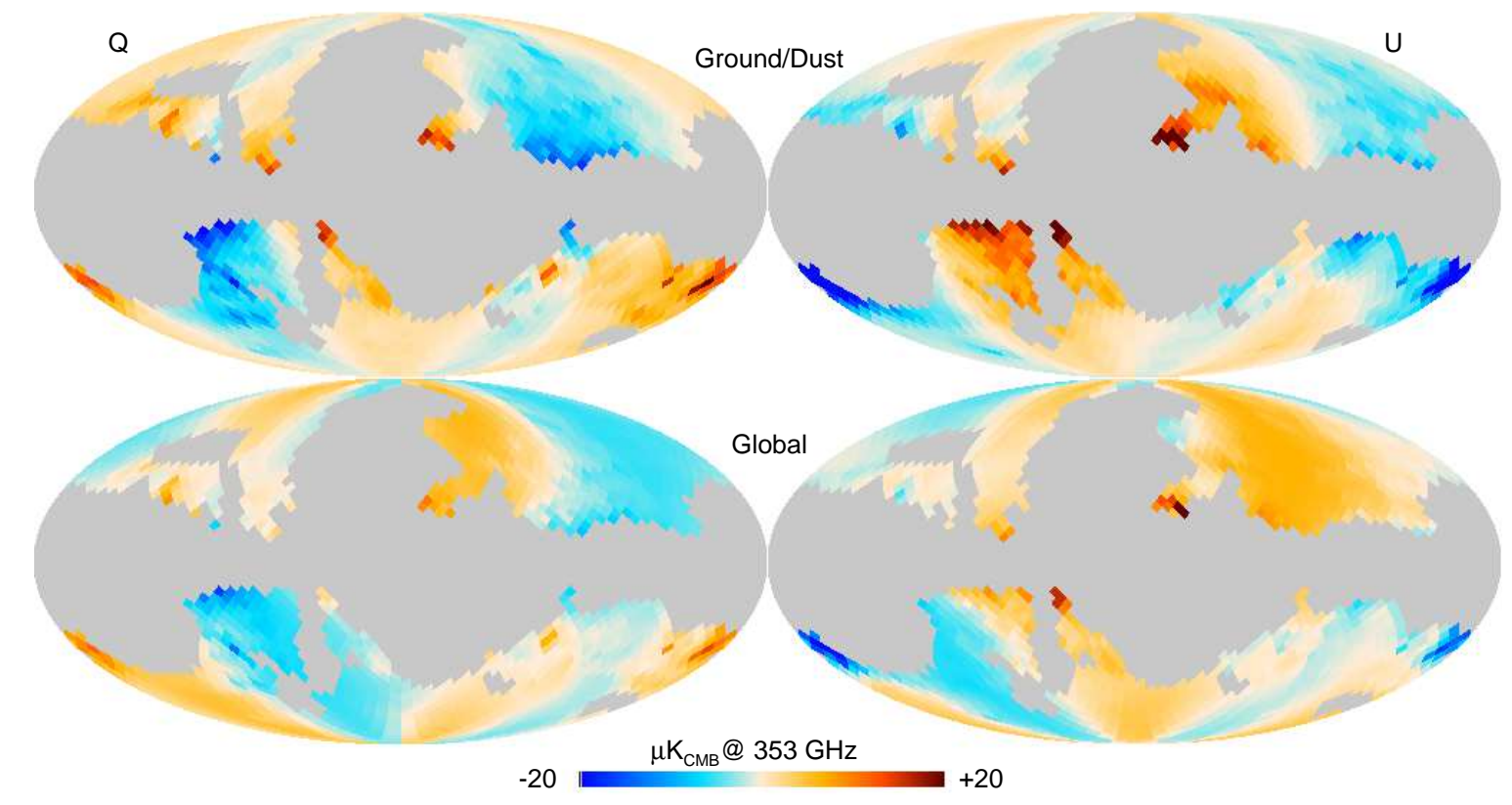

Figure 6. Morphology and magnitude of delivered Planck $2015353 \mathrm{GHz}$ dust template leakage systematic corrections for Stokes Q and U. Top: The ground/dust leakage corrections, which are by default used to correct the delivered Planck $353 \mathrm{GHz}$ full mission map. Bottom: The global leakage correction, which attempts to include both calibration and bandpass mismatch leakage effects, which was made available but not applied to the 2015 products. The global correction has a morphology that is distinguishable from that of the ground/dust correction.

trino masses (Watts et al. 2018).

This research was supported in part by NASA grants NNX16AF28G, NNX17AF34G and by the Canadian Institute for Advanced Research (CIFAR). We acknowledge use of the HEALPix (Gorski et al. 2005) and CAMB (Lewis et al. 2000) packages. This research has made use of NASA's Astrophysics Data System Bibliographic Services. We acknowledge the use of the Legacy Archive for
Microwave Background Data Analysis (LAMBDA), part of the High Energy Astrophysics Science Archive Center (HEASARC). HEASARC/LAMBDA is a service of the Astrophysics Science Division at the NASA Goddard Space Flight Center. We also acknowledge use of the Planck Legacy Archive. Planck is an ESA science mission with instruments and contributions directly funded by ESA Member States, NASA, and Canada.

\section{APPENDIX}

\section{GHZ DUST TEMPLATE}

Full-sky low-resolution $\left(N_{\text {side }}=16\right) 353 \mathrm{GHz}$ Q and U templates are not available from the Planck Legacy Archive. Section 2.3 of Planck Collaboration XI (2016) describes the dust templates used in cleaning the LFI $70 \mathrm{GHz}$ Stokes $\mathrm{Q}$ and $\mathrm{U}$ maps as bandpass-mismatch (BPM) corrected $353 \mathrm{GHz} \mathrm{Q}, \mathrm{U}$ maps degraded to $N_{\text {side }}=16$ using inverse noise weighting. There are however two publicly available options for $353 \mathrm{GHz}$ bandpass mismatch corrections. The first of these is the default option, or "ground/dust leakage correction", which is computed through application of the map-making pipeline to model dust and $\mathrm{CO}$ emission components, in conjunction with transmission coefficients derived by integration of the component spectral response over the ground-based measurements of the bandpass (Planck Collaboration VIII 2016, see Section 7.3.1). A second option, or "global leakage correction", was still in the experimental phase in 2015. As described by Planck Collaboration VIII (2016), this algorithm attempts to simultaneously incorporate bandpass, monopole and calibration leakage into a single correction map set. Maps of both correction types are shown for the region outside the Planck low multipole polarization analysis mask (R1.50) in Figure 6. The inclusion of a CMB dipole signature in the "global" correction is one feature that distinguishes it from the default ground/dust leakage sky pattern. The magnitude of both correction options at $353 \mathrm{GHz}$ is of order $10 \mu \mathrm{K}$ at high Galactic latitudes. Extrapolation of this signal to $70 \mathrm{GHz}$ using the Planck 2015 dust template coefficient of 0.0077 mitigates the correction contribution to a level roughly 3 times smaller than the expected rms of the CMB signal at $N_{\text {side }}=16(0.077$ vs. $0.25 \mu \mathrm{K})$, and Planck Collaboration II (2016) note that changes to the dust template of this order should not have a large effect on the recovered value of $\tau$ from the $70 \mathrm{GHz}$ data.

We attempted to verify which version of the leakage correction was applied to the $353 \mathrm{GHz}$ dust template used to produce the cleaned $70 \mathrm{GHz}$ QU maps delivered in the Planck likelihood. To do this, we solved for the masked 353 $\mathrm{GHz} \mathrm{Q}$, U template contribution, $m_{353}$, based on the cleaned map contained within the likelihood, $m_{c l n}$, and all other known, delivered, components as related by equation 7 of Planck Collaboration XI (2016)

$$
m_{c l n}=\left(1-\alpha_{s}-\alpha_{d}\right)^{-1}\left(m_{70}-\alpha_{s} m_{30}-\alpha_{d} m_{353}\right)
$$




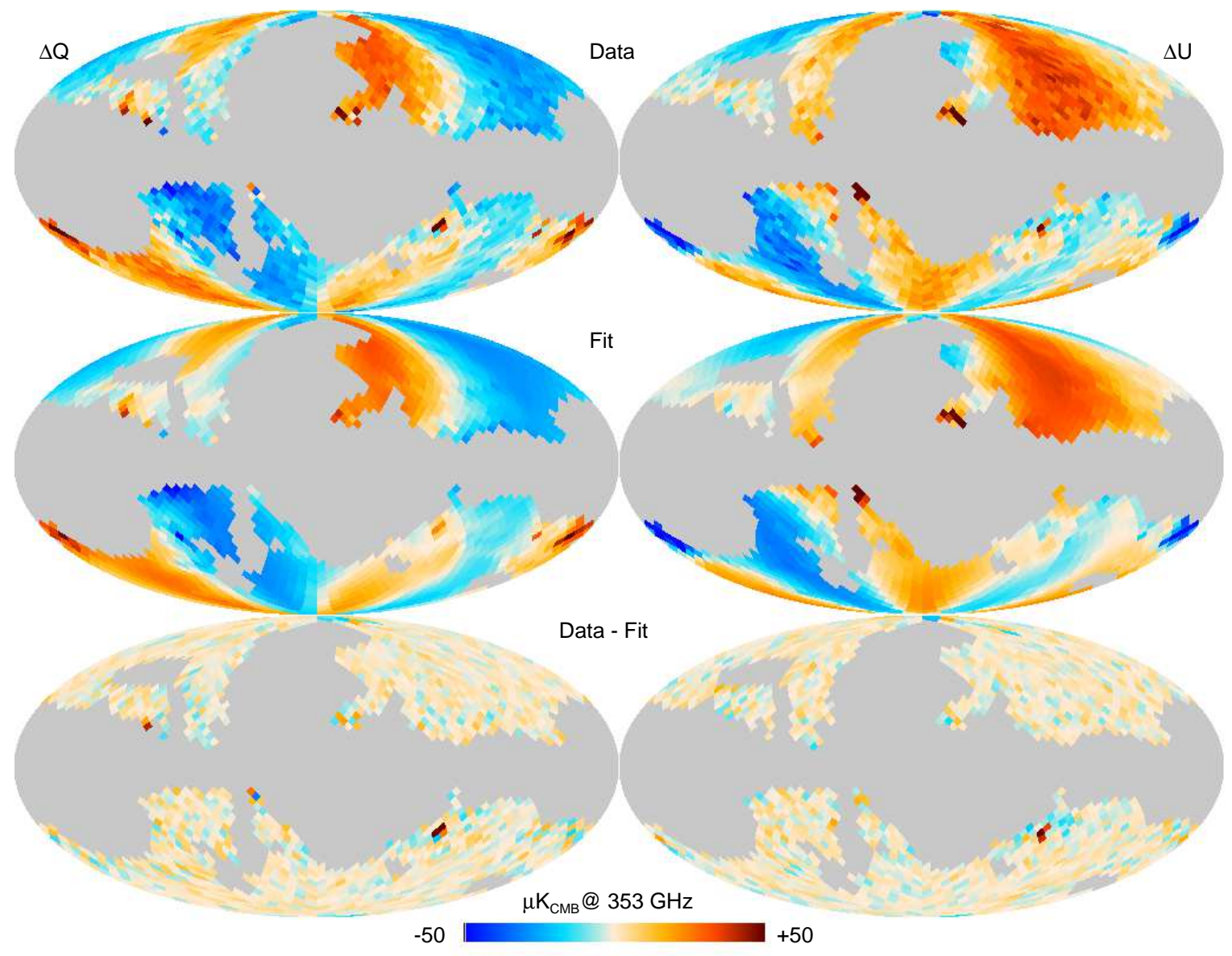

Figure 7. We attempt to verify which BPM correction was applied to the $353 \mathrm{GHz}$ dust template used in the low multipole plc likelihood. Top: the difference between the $353 \mathrm{GHz}$ dust template derived from the plc likelihood (see equation A1) and the delivered $353 \mathrm{GHz}$ map degraded to $N_{\text {side }}=16$. This difference represents the difference between the default ground/dust leakage correction and the leakage correction used for the plc $353 \mathrm{GHz}$ dust template (see text for detail). Middle: a fit to the top row residuals based on a linear combination of the ground/dust leakage template and the global leakage template. The fit is dominated by the global leakage correction term, but with a 5 times larger amplitude. Bottom: residuals of the top row minus the fit in the middle row. The apparent magnitude of the correction applied to the dust template places its contribution at $70 \mathrm{GHz}$ near CMB signal levels, and may affect the determination of $\tau$.

where $m_{70}$ is the observed $70 \mathrm{GHz}[\mathrm{Q}, \mathrm{U}]$ after $\mathrm{BPM}$ correction, $m_{30}$ is observed $30 \mathrm{GHz}[\mathrm{Q}, \mathrm{U}]$ after $\mathrm{BPM}$ correction and acts as the synchrotron template, and $\alpha_{s}, \alpha_{d}$ are the synchrotron and dust template coefficients, respectively, with values 0.063 and 0.0077 as specified in Planck Collaboration XI (2016).

The difference between the default full mission $353 \mathrm{GHz} \mathrm{Q}$ and $\mathrm{U}$ maps, which have been corrected for BPM using the "ground/dust leakage" maps, and the $353 \mathrm{GHz}$ template we derive from the plc cleaned $70 \mathrm{GHz}$ map is shown in the top row of Figure 7. This difference should be approximately zero everywhere if the plc dust template were equivalent to the delivered versions of the corrected full mission $353 \mathrm{GHz}$ QU maps, assuming the baseline uncorrected observed $353 \mathrm{GHz}$ maps are in common: $\Delta m_{353}=m_{353}^{\text {plc }}-m_{353}^{\text {default }}=B P M^{\text {default }}-B P M^{\text {plc }}$. We do expect small high frequency differences in that we did not degrade the $353 \mathrm{GHz}$ map using inverse noise weighting as did Planck, but rather flat weighting after smoothing by a $2^{\circ} \mathrm{FWHM}$ Gaussian. However, the difference predominantly shows large scale coherent signatures of order $30 \mu \mathrm{K}$, whose morphology resembles that of the global correction templates shown in Figure 6, but with a larger amplitude, and cannot be attributed to differences in the map downgrading algorithm. We can fit the difference shown in the top row of this figure with a linear combination of both the ground/dust and global leakage templates: $\Delta m_{353}=A \times B P M^{\text {ground }}+B \times B P M^{\text {global }}$. An unweighted fit returns coefficients $A=-1.00$ and $B=5.36$. The fit result implies that the $353 \mathrm{GHz}$ dust template used to clean the $70 \mathrm{GHz}$ map contained within the plc likelihood was corrected with neither of the delivered leakage correction maps, but rather a correction with global leakage morphology and a 5 times greater amplitude. This is a puzzling and unanticipated result. A $30 \mu \mathrm{K}$ leakage correction at $353 \mathrm{GHz}$ extrapolates roughly to $0.23 \mu \mathrm{K}$ at $70 \mathrm{GHz}$, which is of comparable amplitude to the expected $N_{\text {side }}=16 \mathrm{rms}$ CMB signal for $\tau=0.05$.

Ongoing updates to the HFI calibration pipeline discussed in Planck Collaboration Int. XLVI (2016) indicate that the global leakage algorithm may ultimately provide a more realistic assessment of the BPM correction. However, the delivered 2015 global leakage template is described as only preliminary, and, as discussed here, its amplitude is uncertain. We found no reason to favor one template over the other and thus included both plc and default options 
for the $353 \mathrm{GHz}$ polarized dust emission template in our fits for $\tau$; see Table 2 and Section 3 .

\section{REFERENCES}

Addison, G. E., Huang, Y., Watts, D. J., et al. 2016, ApJ, 818, 1325

Addison, G. E., Watts, D. J., Bennett, C. L., et al. 2018, ApJ, 853,1195

Allison, R., Caucal, P., Calabrese, E., Dunkley, J., \& Louis, T. 2015, Phys. Rev. D, 92, 1235351

Aylor, K., Hou, Z., Knox, L., et al. 2017, ApJ, 850, 1015

Bennett, C. L., Larson, D., Weiland, J. L., et al. 2013, ApJS, 208, $201,2,4,4$

Boyle, A., \& Komatsu, E. 2018, J. Cosmology Astropart. Phys., 3, 0351

Calabrese, E., Alonso, D., \& Dunkley, J. 2017, Phys. Rev. D, 95, 0635041

Fan, X., Strauss, M. A., Becker, R. H., et al. 2006, AJ, 132, 1171

Finkbeiner, D. P., Davis, M., \& Schlegel, D. J. 1999, ApJ, 524, 8671

Foreman-Mackey, D., Hogg, D. W., Lang, D., \& Goodman, J. 2013, PASP, 125, 3062

Gorski, K. M., Hivon, E., Banday, A. J., et al. 2005, ApJ, 622, 7592,5

Gunn, J. E., \& Peterson, B. A. 1965, ApJ, 142, 16331

Hinshaw, G., Larson, D., Komatsu, E., et al. 2013, ApJS, 208, 19 1

Jarosik, N., Barnes, C., Greason, M. R., et al. 2007, ApJS, 170, $263 \quad 4.1$
Jarosik, N., Bennett, C. L., Dunkley, J., et al. 2011, ApJS, 192, 144

Lewis, A., Challinor, A., \& Lasenby, A. 2000, ApJ, 538, 4735

Page, L., Hinshaw, G., Komatsu, E., et al. 2007, ApJS, 170, 335

$2,2,3,4,4.2$

Planck Collaboration I. 2016, A\&A, 594, A1 2

Planck Collaboration II. 2016, A\&A, 594, A2 1, 4.1, 4.2, A

Planck Collaboration III. 2014, A\&A, 571, A3 4.1

Planck Collaboration Int. XLVI. 2016, A\&A, 596, A107 1, 1, 4.4, 5, A

Planck Collaboration Int. XLVII. 2016, A\&A, 596, A108 1

Planck Collaboration VIII. 2016, A\&A, 594, A8 1, A

Planck Collaboration X. 2016, A\&A, 594, A10 1, 4, 4, 4.3, 5

Planck Collaboration XI. 2016, A\&A, 594, A11 1, 1, 2, 3, 5, A, A, A

Planck Collaboration XIII. 2016, A\&A, 594, A13 1, 2, 2

Planck Collaboration XXV. 2016, A\&A, 594, A25 4, 5

Pryke, C., Halverson, N. W., Leitch, E. M., et al. 2002, ApJ, 568, $46 \quad 1$

Tegmark, M., \& de Oliveira-Costa, A. 2001, Phys. Rev. D, 64, 0630012

Watts, D. J., Wang, B., Ali, A., et al. 2018, ArXiv e-prints, arXiv:1801.01481 1, 5

Wehus, I. K., \& Eriksen, H. K. 2017, ArXiv e-prints, arXiv:1712.10223 4

Zaldarriaga, M., Spergel, D. N., \& Seljak, U. 1997, ApJ, 488, 11 